\title{
Usefulness and Future Prospects of Confocal Laser Endomicroscopy for Gastric Premalignant and Malignant Lesions
}

\author{
Sang Kil Lee \\ Division of Gastroenterology, Department of Internal Medicine, Yonsei Institute of Gastroenterology, Yonsei University College of Medicine,
} Seoul, Korea

Confocal laser endomicroscopy (CLE) is a new technology enabling endoscopists to visualize tissue at the cellular level. CLE has the fundamental potential to provide a histologic diagnosis, and may theoretically replace or reduce the need for performing biopsy for histology. The clinical benefits of CLE are more obvious in esophageal disease, including Barrett's esophagus. Currently, this technology has been adapted to the diagnosis and surveillance of Barrett's esophagus and related neoplasia. Standard white light endoscopy is the primary tool for gastric cancer screening. Currently, the only method available to precisely diagnose these lesions is upper endoscopy with an appropriate biopsy. A recent study showed that CLE could characterize dysplasia or cancer and identify the risk factors for gastric cancer, such as intestinal metaplasia and the presence of Helicobacter pylori in vivo, although fewer studies on CLE were performed on the stomach than on Barrett's esophagus and other esophageal diseases. However, the application of CLE to routine clinical endoscopy continues to be refined. This review focused on the usefulness and future prospects of CLE for gastric premalignant and malignant lesions. Clin Endosc 2015;48:511-515

Key Words: Confocal laser endomicroscopy; Stomach neoplasms; Gastric intestinal metaplasia

\section{INTRODUCTION}

Endoscopy is an essential procedure for the diagnosis of a gastrointestinal (GI) disease. Biopsy and histology were the gold standards for final diagnosis. However, detection of premalignant and early malignant lesions in the inflamed stomach and intestine remains difficult. In recent years, a range of innovative techniques have entered the endoscopic arena due to their ability to enhance contrast and magnify diseased tissue regions at the cellular level, beyond the ability of standard white-light endoscopy (WLE) equipment. Confocal laser endomicroscopy (CLE) is a technology that

Received: October 21, 2015 Revised: November 16, 2015

Accepted: November 17, 2015

Correspondence: Sang Kil Lee

Division of Gastroenterology, Department of Internal Medicine, Yonsei Institute of Gastroenterology, Yonsei University College of Medicine, 50-1 Yonsei-ro, Seodaemun-gu, Seoul 03722, Korea

Tel: +82-2-2228-1996, Fax: +82-2-393-6884, E-mail: sklee@yuhs.ac

cc This is an Open Access article distributed under the terms of the Creative Commons Attribution Non-Commercial License (http://creativecommons.org/ licenses/by-nc/3.0) which permits unrestricted non-commercial use, distribution, and reproduction in any medium, provided the original work is properly cited. enables microscopic views $(\times 1,000)$ of the mucosa in real time during endoscopy. The technology can be used either via a single endoscope-based system (eCLE; OptiScan, Notting Hill, Australia) or via probe-based CLE (pCLE, Cellvizio; Mauna Kea Technologies, Paris, France). ${ }^{1}$ CLE is one of the newest advancements in diagnostic endoscopy, and is a highly promising technique for investigating the mucosal surface and the immediate subsurface areas. Cell structures and tissue morphological characteristics can be visualized to a maximum depth of $250 \mu \mathrm{m}$. ${ }^{1}$ This technique aims to achieve few or no biopsies in regions of interest via multiple optical biopsies. The usefulness of CLE is evaluated to shift the focus from random to targeted biopsies.

Gastric cancer is known to develop either de novo or from precancerous lesions. Helicobacter pylori infection progresses from non-atrophic gastritis to chronic atrophic gastritis and from intestinal metaplasia to dysplasia and intestinal non-cardia adenocarcinoma. ${ }^{2}$ To the best of our knowledge, Japan and Korea are the only countries with ongoing nationwide organized gastric cancer screening programs. Standard WLE is the primary tool for screening. Currently, the only method 
available to precisely diagnose these lesions is upper endoscopy with an appropriate biopsy. In Asian countries including Korea and Japan, early gastric cancer (EGC) is detected by WLE; however, identification of EGC is difficult because some lesions are too small to recognize and characterize. Metaplastic lesions in the background make EGC difficult to find. In this review, we will discuss the role and future prospects of CLE that enable characterization of neoplastic and preneoplastic lesions better than conventional endoscopy.

\section{TYPES OF CLE AND THEIR CHARACTERISTICS}

CLE is currently performed with two systems, eCLE and pCLE. eCLE was launched earlier than pCLE, but is no longer marketed. Both systems use blue laser light $(488 \mathrm{~nm}$ ) for excitation, and require contrast. Fluorescein is mostly used as the intravenous (IV) contrast agent. Compared to eCLE, pCLE can pass through any type of conventional endoscope, including state-of-the-art high definition endoscopes. ${ }^{3}$ eCLE offers a wider field of view, the ability to vary the depth of imaging from 0 to $250 \mu \mathrm{m}$, and slightly better lateral resolution. Advantages of pCLE are the versatility of probe system that can be passed via any endoscope, or even needles, and more rapid image acquisition (12 frames/sec vs. 0.8 to 1.6 frames/ sec), which allows imaging of the blood flow in vivo. ${ }^{4}$

Both systems are basically similar, but differ in details such as image resolution, field of view, and depth of imaging. Because researchers and clinicians use only one of these systems, the opportunity to compare the two is rare. To our knowledge, close comparison of the two systems in clinical practice is not reported to date; therefore, usability of the same diagnostic classification for specific diseases by both systems is unclear. Moreover, CLE is classified according to two criteria: the Mainz classification and the Miami classification. The Mainz classification distinguishes neoplastic from hyperplastic polyps of the colon based on a dark, irregularly thickened epithelial layer characteristic of epithelial dysplasia. ${ }^{5}$ The Miami classification was similarly established for pCLE to distinguish normal and pathological GI conditions. ${ }^{3}$ During the review of articles and analysis of results, the types of system used should be considered. Because eCLE has been discontinued, pCLE is likely to dominate the market. Both systems have inherent limitations compared with WLE, including a very small field of view, high cost of the technology, long learning curve, and extra time needed to view the images during endoscopy.

\section{CLE FOR DIAGNOSIS OF H. PYLORI IN VIVO}

H. pylori is an accepted major etiological factor in gastric diseases, such as chronic gastritis, peptic ulcer, and gastric carcinoma. ${ }^{6,7}$ Kiesslich et al. ${ }^{8}$ first reported in 2004 that $H$. pylori was detected in vivo by eCLE in a single patient. The study suggested that CLE permits immediate imaging of live bacteria in the stomach, and thus can be used for diagnosis of $\mathrm{H}$. pylori infection during ongoing gastroscopy. eCLE was performed using topical acriflavine. Chinese researchers reproduced this using eCLE in 2010. ${ }^{9}$ In this study, the authors concluded that eCLE findings, including white spots, neutrophils, and microabscesses, showed positive association with H. pylori diagnosis. To our knowledge, these studies were the first to show that CLE can detect bacteria; however, this indication for CLE is very limited because of the fact that eCLE is no longer available and because acriflavine accumulates in the nuclei, leading to a concern of potential mutagenic risk. Wang et al. ${ }^{10}$ analyzed eCLE features of gastritis caused by $H$. pylori infection. They concluded that eCLE can accurately show the histological severity of gastritis associated with $H$. pylori infection. To our knowledge, this was the first study to show gastropathy associated with $H$. pylori infection using eCLE. After several initial studies, no further study was reported on this subject. Moreover, no studies compared other methods for detecting $H$. pylori. The possibility of detecting either localization or dispersion of $H$. pylori residue in the stomach and the role of CLE in detecting $H$. pylori will be clinically limited due to CLE's narrow range of examination.

\section{eCLE FOR DIAGNOSIS OF CANCER AND PREMALIGNANT CONDITIONS}

To the best of our knowledge, the first trial of scanning for gastric cancer was reported by Japanese researchers in 2006. A prototype of an eCLE system with acriflavine was used and the possibility of diagnosis of malignancy in more than half the cases was reported. ${ }^{11}$ The next study on human gastric cancer, also performed in Japan, was conducted using eCLE and $500 \mathrm{mg}$ fluorescein sodium IV as a contrast agent. ${ }^{12}$ eCLE had an accuracy of $94 \%$ to $96 \%$ for the diagnosis of malignancy among 27 patients with EGC, when compared directly with histological biopsies. IV fluorescein had pros and cons compared to acriflavine. IV fluorescein allows visualization of ductal structures and deeper levels; however, acriflavine can stain the surface layer of mucosa intensely, allowing identification of the cell structure and nuclei, but the deeper layer is not revealed. In this study, the accuracy of diagnosis of gastric cancer by eCLE in two different pathologists was $94.2 \%$, and $96.2 \%$, respectively. The accuracy decreased substantially when poor images and inaccessible lesions were included. The characterization of cancer in upper GI mucosa using eCLE with fluorescein sodium was subsequently studied in China. ${ }^{13}$ 
In this study, eight gastric cancer patients, six with superficial esophageal carcinoma, and 10 normal individuals were evaluated; however, eCLE could provide clear images of gland architecture and microvascular structures, and made it possible to differentiate early carcinoma and normal mucosa. ${ }^{13}$

In 2008, Zhang et al. ${ }^{14}$ tried to establish classification of the gastric pit patterns using eCLE and the relationship between the patterns and relevant histopathologic findings. ${ }^{15}$ They reported seven types of gastric pit patterns. Type A (round pits) represented normal mucosa with fundic glands; type B (noncontinuous, short, rod-like) corporal mucosa with histologic gastritis; type C (continuous, short, rod-like) normal mucosa with pyloric glands; type $\mathrm{D}$ (elongated and tortuous, branch-like) antral mucosa with histologic gastritis; type $\mathrm{E}$ (the number of pits decreasing and pits prominently dilating) chronic atrophic gastritis; and type F (villus-like appearance, interstitium in the center and goblet cells appearing) intestinal metaplasia. Type G was subdivided into G1 (normal pits disappearing, with the appearance of diffusely atypical cells), which represented signet ring cell carcinoma and poorly differentiated adenocarcinoma, and G2 (normal pits disappearing with the appearance of atypical glands), which represented differentiated tubular adenoma. To the best of our knowledge, this study is the first to systematically classify pit patterns and correlate them with disease spectrums in the stomach. The sensitivity and specificity of the type $G$ pattern for predicting gastric cancer were $90.0 \%$ and $99.4 \%$. Although this study introduced the systematic classification of eCLE findings corresponding to histologic findings, interobserver and intraobserver variability was not evaluated.

In 2010 , Li et al. ${ }^{16}$ tried to determine the feasibility of eCLE using fluorescein sodium for identification and grading of gastric intraepithelial neoplasia (GIN). Thirty-three patients with histologically confirmed normal mucosa $(n=4)$ and non-neoplastic ( $n=12)$ and neoplastic ( $n=17$; including 12 low-grade IN and 5 high-grade intraepithelial neoplasia [HGIN]) lesions were evaluated to establish the diagnostic criteria for GIN (Table 1). These criteria were evaluated in 75 consecutive patients. The sensitivity, specificity, and positive and negative likelihood ratios of eCLE diagnosis for GIN were $77.8 \%, 81.8 \%, 4.28$, and 0.27 , respectively. The mean $\kappa$ value for interobserver agreement for the diagnosis of GIN was 0.70 among endoscopists and 0.71 in a comparison between the endoscopist and GI pathologist. However, not all parameters that represent the accuracy of eCLE for GIN were as positive as eCLE for gastric intestinal metaplasia (GIM). Detailed examination of the cell nuclei is mandatory to determine GIN histologically, but a fundamental limitation of CLE with fluorescein is the inability to define the nuclear structure. This can hinder precise CLE diagnosis of GIN, resulting in a reduced diagnostic accuracy compared to GIM.

In the largest study published on the use of eCLE with fluorescein sodium for the characterization of gastric superficial cancerous lesions, 182 patients were enrolled in phase I to establish morphologic criteria for gastric superficial cancerous lesions, and 1,786 patients were enrolled in phase II for prospective validation. ${ }^{17} \mathrm{CLE}$ criteria for cancer/HGIN lesions were irregularity in glandular size and shape, disorganized or destroyed pits and glands, irregular cells with disordered appearance, severe stratification, loss of cell polarity, and irregular shape and caliber of vessels (Table 1). The study was then used to establish a simplified two-tiered CLE classification of

Table 1. Endomicroscopic Classification of Gastric Lesions ${ }^{16,17}$

\begin{tabular}{|c|c|c|c|}
\hline & Gland architecture & Cell morphology & Vessel architecture \\
\hline Normal architecture & $\begin{array}{l}\text { Regularly ranged glands, with round } \\
\text { (fundic glands) or continuous short } \\
\text { rod-like (pyloric glands) pits }\end{array}$ & $\begin{array}{l}\text { Homogeneous epithelial cells } \\
\text { with normal polarity }\end{array}$ & $\begin{array}{l}\text { Honeycomb-like (gastric } \\
\text { body) or coil-shaped (gastric } \\
\text { antrum) }\end{array}$ \\
\hline $\begin{array}{l}\text { Non-neoplastic lesion } \\
\text { (not IM, not IN) }\end{array}$ & $\begin{array}{l}\text { Good polarity with elongated pits } \\
\text { Homogeneous in size and epithelial } \\
\text { heights }\end{array}$ & $\begin{array}{l}\text { Good cell polarity: regularly } \\
\text { ranged epithelial cells, uniform } \\
\text { in size and shape }\end{array}$ & $\begin{array}{l}\text { Honeycomb-like or coil- } \\
\text { shaped, no or mild increase } \\
\text { in the capillaries number }\end{array}$ \\
\hline $\mathrm{IM}$ & Villous appearance & $\begin{array}{l}\text { Large black 'goblet cells'; slender } \\
\text { tall, and bright 'absorptive' cells }\end{array}$ & $\begin{array}{l}\text { Normal calibre, honey-comb } \\
\text { like or coil-shaped }\end{array}$ \\
\hline IN & $\begin{array}{l}\text { Impaired gland polarity: crowded } \\
\text { glands with variable degrees of } \\
\text { intraluminal folding, glandular } \\
\text { budding and branching } \\
\text { Irregular in size and epithelial heights }\end{array}$ & $\begin{array}{l}\text { Abnormal cell polarity: mild to } \\
\text { severe irregularity of cellular } \\
\text { arrangement } \\
\text { Hyperdense epithelial cells with } \\
\text { increased stratification }\end{array}$ & $\begin{array}{l}\text { Dilated and distorted } \\
\text { appearance }\end{array}$ \\
\hline Cancer & $\begin{array}{l}\text { Loss of gland polarity: disorganised or } \\
\text { destroyed }\end{array}$ & $\begin{array}{l}\text { Loss of cell polarity: irregular } \\
\text { and variable in size, disordered } \\
\text { appearance }\end{array}$ & $\begin{array}{l}\text { Increased calibre and irregular } \\
\text { in size and shape }\end{array}$ \\
\hline
\end{tabular}

IM, intestinal metaplasia; IN, intraepithelial neoplasia. 
Table 2. Simple Tow-Tiered Confocal Laser Endomicroscopy Classification for Gastric Superficial Lesions ${ }^{17}$

\begin{tabular}{lcc}
\hline Feature & \multicolumn{1}{c}{ Non-cancerous lesions } & \multicolumn{1}{c}{ Cancer/HGIN lesions } \\
\hline Architecture & $\begin{array}{c}\text { Orderly ranged glands with regular pit patterns, or mildly } \\
\text { heterogeneous in arrangement and distribution }\end{array}$ & $\begin{array}{c}\text { Irregularity in glandular size and shape; disorganised or } \\
\text { destroyed pits and glands }\end{array}$ \\
Cells & $\begin{array}{c}\text { Regular in shape and size; mildly increase in epithelial } \\
\text { stratification; normal cell polarity }\end{array}$ & $\begin{array}{c}\text { Irregular cells with disordered appearance; severe stratifi- } \\
\text { cation; loss of cell polarity }\end{array}$ \\
\hline
\end{tabular}

HGIN, high grade intraepithelial neoplasia.

non-cancerous lesions and EGC/HGIN lesions (Table 2). Using these criteria, eCLE had higher sensitivity (88.9\%), specificity (99.3\%), and accuracy (98.8\%) for the diagnosis of gastric superficial cancer/HGIN lesions than WLE (sensitivity, 72.2\%; specificity, $95.1 \%$; and accuracy, $94.1 \%){ }^{17}$

GIM is a risk factor that leads to the development of intestinal-type gastric cancer. A group of 28 Chinese patients with known intestinal metaplasia underwent eCLE, and CLE criteria for diagnosis of GIM were developed. In addition, 53 consecutive patients with known or suspected GIM were prospectively evaluated. The sensitivities of conventional endoscopy and CLE for GIM were $36.88 \%$ versus $98.13 \%$, and the specificities were $91.59 \%$ versus $95.33 \%$, respectively. The $\kappa$ value for the correlation with histological findings was 0.25 for conventional endoscopy versus 0.94 for CLE. ${ }^{15}$ Furthermore, trials were conducted to identify and grade intestinal metaplasia and atrophic gastritis that were confirmed as precancerous lesions of gastric cancer by eCLE. CLE showed the distinct features of intestinal metaplasia, and when used with targeted biopsies, it is superior to WLE with standard biopsies for the detection and surveillance of GIM. ${ }^{18}$

Recently, Liu et al. ${ }^{19}$ reported a new application of eCLE for grading of atrophic gastritis. In this study, eCLE was compared to chromoendoscopy and narrow band imaging (NBI) for diagnosing atrophic gastritis. eCLE had a higher sensitivity, specificity, and accuracy compared with NBI and CE, which were equivalent for the diagnosis of atrophic gastritis. The sensitivity and specificity of eCLE for diagnosing nonmetaplastic atrophy were $86.76 \%$ and $91.89 \%$, respectively, and for metaplastic atrophy were $91.94 \%$ and $96.86 \%$, respectively. Both interobserver and intraobserver agreement were high.

\section{pCLE FOR DIAGNOSIS OF CANCER AND PREMALIGNANT CONDITIONS}

Compared to eCLE, pCLE has rarely been studied for gastric lesions. Pittayanon et al. ${ }^{20}$ assessed the diagnostic yield of magnifying endoscopy with flexible spectral imaging color enhancement (ME-FICE) and pCLE with fluorescein sodium for GIM. The overall criteria from ME-FICE plus pCLE provided the highest sensitivity, specificity, positive predictive value, negative predictive value, and accuracy at $96 \%, 90 \%$,
$86 \%, 97 \%$, and $92 \%$, respectively. This study showed that a combination of broad-field (red-flag) techniques with pCLE would be a promising tool for screening gastric lesions. An additional study that compared the diagnostic accuracy of autofluorescence imaging (AFI), magnifying NBI, and pCLE with WLE for the diagnosis of GIM, using histology as the gold standard. ${ }^{21}$ pCLE was more accurate than AFI and WLE for the diagnosis of GIM.

\section{CLE APPLICATION FOR ENDOSCOPIC RESECTION AND ENDOSCOPIC SUBMUCOSAL DISSECTION}

Korean researchers compared the accuracy of PCLE and WLE for EGCs scheduled for endoscopic submucosal dissection (ESD). ${ }^{22}$ For complete ESD, correct pre-ESD histologic diagnosis is mandatory. Furthermore, multiple pre-ESD biopsies can seriously hamper subsequent ESD because inflammatory change thereafter often results in fibrosis of the submucosal layer. In this study, combining pCLE with conventional WLE significantly improved the accuracy of pre-ESD histologic diagnosis. This study showed that PCLE has a strong beneficial role in the cases of EGC that are candidates for subsequent ESD. The same group also studied eCLE for this purpose and demonstrated similar results. ${ }^{23}$ A Study on the use of pCLE to examine the delineation of margins of gastric epithelial tumors with ESD is being conducted by Korean researchers.

\section{CONCLUSIONS}

Although conventional histology is still the gold standard for diagnosis, endoscopists can take advantage of CLE. Based on the published papers, CLE shows the clinical benefits of targeted biopsy by aiding selection of appropriate biopsy sites for suspicious lesions, which possibly increases the rate of positive biopsy results and reduces the number of biopsies. However, currently available studies on CLE for gastric lesions are very limited because of a variety of factors. Most studies deal with detection of GIM and atrophy by eCLE. The clinical value of characterization of IM and atrophy by CLE is not high because of its limited area of examination, despite the ability of CLE to replace the laborious Sydney System 5-biop- 
sy protocol. Further studies including coordination with redflag technologies and molecular imaging are needed to extend CLE applications into clinical areas.

\section{Conflicts of Interest}

The author has no financial conflicts of interest.

\section{REFERENCES}

1. Committee AT. Confocal laser endomicroscopy. Gastrointest Endosc 2014;80:928-938.

2. Kapadia CR. Gastric atrophy, metaplasia, and dysplasia: a clinical perspective. J Clin Gastroenterol 2003;36(5 Suppl):S29-S36.

3. Wallace M, Lauwers GY, Chen Y, et al. Miami classification for probebased confocal laser endomicroscopy. Endoscopy 2011;43:882-891.

4. Arya AV, Yan BM. Ultra high magnification endoscopy: is seeing really believing? World J Gastrointest Endosc 2012;4:462-471.

5. Kiesslich R, Burg J, Vieth M, et al. Confocal laser endoscopy for diagnosing intraepithelial neoplasias and colorectal cancer in vivo. Gastroenterology 2004;127:706-713.

6. Uemura N. The magnitude of association between Helicobacter pylori infection and the development of gastric cancer. Scand J Gastroenterol 2002;37:869-870.

7. Goldblum JR. Inflammation and intestinal metaplasia of the gastric cardia: Helicobacter pylori, gastroesophageal reflux disease, or both. Dig Dis 2000;18:14-19.

8. Kiesslich R, Goetz M, Burg J, et al. Diagnosing Helicobacter pylori in vivo by confocal laser endoscopy. Gastroenterology 2005;128:2119-2123.

9. Ji R, Li YQ, Gu XM, Yu T, Zuo XL, Zhou CJ. Confocal laser endomicroscopy for diagnosis of Helicobacter pylori infection: a prospective study. J Gastroenterol Hepatol 2010;25:700-705.

10. Wang P, Ji R, Yu T, et al. Classification of histological severity of Helicobacter pylori-associated gastritis by confocal laser endomicroscopy. World J Gastroenterol 2010;16:5203-5210.

11. Kakeji Y, Yamaguchi S, Yoshida D, et al. Development and assessment of morphologic criteria for diagnosing gastric cancer using confocal endomicroscopy: an ex vivo and in vivo study. Endoscopy 2006;38:886-
890.

12. Kitabatake S, Niwa Y, Miyahara R, et al. Confocal endomicroscopy for the diagnosis of gastric cancer in vivo. Endoscopy 2006;38:1110-1114.

13. Liu $\mathrm{H}, \mathrm{Li} \mathrm{YQ}$, Yu T, et al. Confocal endomicroscopy for in vivo detection of microvascular architecture in normal and malignant lesions of upper gastrointestinal tract. J Gastroenterol Hepatol 2008;23:56-61.

14. Zhang JN, Li YQ, Zhao YA, et al. Classification of gastric pit patterns by confocal endomicroscopy. Gastrointest Endosc 2008;67:843-853.

15. Guo YT, Li YQ, Yu T, et al. Diagnosis of gastric intestinal metaplasia with confocal laser endomicroscopy in vivo: a prospective study. Endoscopy 2008;40:547-553.

16. Li Z, Yu T, Zuo XL, et al. Confocal laser endomicroscopy for in vivo diagnosis of gastric intraepithelial neoplasia: a feasibility study. Gastrointest Endosc 2010;72:1146-1153.

17. Li WB, Zuo XL, Li CQ, et al. Diagnostic value of confocal laser endomicroscopy for gastric superficial cancerous lesions. Gut 2011;60:299-306.

18. Li Z, Zuo XL, Yu T, et al. Confocal laser endomicroscopy for in vivo detection of gastric intestinal metaplasia: a randomized controlled trial. Endoscopy 2014;46:282-290.

19. Liu T, Zheng H, Gong W, Chen C, Jiang B. The accuracy of confocal laser endomicroscopy, narrow band imaging, and chromoendoscopy for the detection of atrophic gastritis. J Clin Gastroenterol 2015;49:379-386.

20. Pittayanon R, Rerknimitr R, Wisedopas N, et al. Flexible spectral imaging color enhancement plus probe-based confocal laser endomicroscopy for gastric intestinal metaplasia detection. J Gastroenterol Hepatol 2013;28:1004-1009.

21. Lim LG, Yeoh KG, Srivastava S, Chan YH, Teh M, Ho KY. Comparison of probe-based confocal endomicroscopy with virtual chromoendoscopy and white-light endoscopy for diagnosis of gastric intestinal metaplasia. Surg Endosc 2013;27:4649-4655.

22. Bok GH, Jeon SR, Cho JY, et al. The accuracy of probe-based confocal endomicroscopy versus conventional endoscopic biopsies for the diagnosis of superficial gastric neoplasia (with videos). Gastrointest Endosc 2013;77:899-908.

23. Jeon SR, Cho WY, Jin SY, Cheon YK, Choi SR, Cho JY. Optical biopsies by confocal endomicroscopy prevent additive endoscopic biopsies before endoscopic submucosal dissection in gastric epithelial neoplasias: a prospective, comparative study. Gastrointest Endosc 2011;74:772-780. 